\title{
Body mass index in young men in Switzerland after the national shutdowns during the COVID-19 pandemic - Results from a cross- sectional monitoring study at population level since 2010
}

Samuel Meili, MMed ${ }^{1,2}$; Marek Brabec, PhD ${ }^{3}$; Frank Rühli, MD PhD ${ }^{1}$; Thomas W. Buehrer, MD ${ }^{4}$; Nejla Gültekin, MD ${ }^{4,5}$; Zeno Stanga, MD ${ }^{1,5}$; Nicole Bender, MD PhD ${ }^{2,7}$; Kaspar Staub, PhD ${ }^{2,6,7 *}$; Emilie Reber, $\operatorname{PhD}{ }^{1,5 *}$

${ }^{1}$ Department of Diabetes, Endocrinology, Nutritional Medicine and Metabolism, University Hospital and University of Bern, Switzerland

${ }^{2}$ Institute of Evolutionary Medicine, University of Zurich, Zurich, Switzerland

${ }^{3}$ Institute of Computer Science of the Czech Academy of Sciences, Prague, Czech Republic

${ }^{4}$ Swiss Armed Forces, Medical Services, Ittigen, Switzerland

${ }^{5}$ Centre of Competence for Military and Disaster Medicine, Swiss Armed Forces, Ittigen, Switzerland

${ }^{6}$ Zurich Center for Integrative Human Physiology (ZIHP), University of Zurich, Switzerland

${ }^{7}$ Swiss School of Public Health SSPH+

* These authors contributed equally: Shared last-authorship

Address for correspondence including e-mail address

PD Dr. Kaspar Staub, Institute of Evolutionary Medicine, University of Zurich, Winterthurerstrasse 190, CH8057 Zurich, Switzerland, kaspar.staub@iem.uzh.ch, +41 446350513

Word count: 2’856 


\begin{abstract}
Background: Due to the COVID-19 pandemic, the Swiss Federal Council imposed a shutdown twice, which may have changed dietary and physical activity habits. On the question of weight change during the pandemic, there is little information based on measured weight data. In this study, we investigate whether the body mass index (BMI) of young Swiss men conscripted after the two shutdown periods in Spring and Fall 2020 differed from the conscripts examined before the shutdowns.
\end{abstract}

Methods: We are analysing body measurements of young Swiss men taken during mandatory recruitment for the Swiss Armed Forces on the cross-sectional (not individual longitudinal) monitoring level and across weeks of conscription between January 2010 and July $2021(n=373,016)$. These cross-sectional data allow for continuous health monitoring of young men almost at the population level (coverage $>90 \%$ of recruitment relevant age categories). For statistical modelling, we use GAM (Generalized Additive Model framework.

Results: We showed that the BMI of conscripts who were examined in the 15 weeks after the two shutdowns in Spring and Autumn 2020 was not or only weakly different from baseline. Sensitivity analyses showed that this conclusion also holds if assessing the BMI distribution or the prevalence of overweight and/or obesity. The GAM models further showed strong effects of individual and area-based SEP as well as age on BMI.

Conclusion: Our results suggest that any lifestyle changes during the pandemic in young men may have been too modest to be clearly reflected in body weight (suggesting small or none effect). However, longitudinal data and/or data on women, children, or the elderly may lead to different conclusions.

Keywords: BMI; Obesity; Monitoring; Lockdown; swiss Conscription; GAM 


\section{Introduction}

In the wake of the COVID-19 pandemic in Spring 2020, the Federal Council decided on the first far-reaching shutdown on March 16, 2020, which was relaxed in several steps between April and June 2020.(1) Due to this first national shutdown, the lifestyle of the Swiss population changed markedly. On the one hand, physical activity behaviour was affected through staying home recommendations, home offices, suspension of schools, closed sports facilities and gyms, etc. On the other hand, eating habits, work environments, and mental wellbeing might also have changed, as has been documented for other countries.(2) These affected lifestyle factors are all well known to be associated with weight gain, overweight, and obesity. In autumn 2020, far-reaching measures were again taken by the Federal Council to limit the peak of the second COVID-19 wave. Nationwide mobility tracking in Switzerland has shown a striking reduction in the daily travelled distance during both periods.(3) Younger people were affected in particular, as they are the most mobile group of the population.(3) At present, it is unclear how these two shutdown periods have affected the health of the general population in Switzerland, especially concerning body weight. Based on the literature (4-7) nutritional status could have been influenced in two directions: increased outdoor activities combined with healthier nutrition (homemade, "slow food") may have led to less overweight. However, it is also conceivable that body weight was adversely influenced by increased immobility, unhealthier food, and psychological stress.

Since 2020, there has been a steady increase in the number of studies addressing this issue based on electronic surveys that capture the health and nutritional status of individuals based on self-reported information during home confinement. Two recent review articles summarize the evidence about weight gain during shutdowns in Spring 2020 based on surveys from various countries. The first systematic review and meta-analysis based on 59,000 people from 32 different countries documents weight gain regardless of age group.(8) The second review also highlights different clusters of weight change, with people with high baseline BMI and with low education level being particularly affected by weight gain.(9) For Switzerland, a recent survey based on selfreported weight information showed that between 2019 and 2021, the age group between 45-64 years gained more than four times as much weight as the younger age groups.(10)

In general, there is little information on this question of weight change during the pandemic based on measured weight data. Our study is intended to change this for Switzerland. We are analysing body measurements of young Swiss men taken during mandatory recruitment for the Swiss Armed Forces, which allow for continuous 
health monitoring of young men almost at the population level (it is a unique feature of our study that it circumvents essentially all potential problems with sample selection by investigating almost all conscriptable individuals). On the cross-sectional (not individual longitudinal) monitoring level and across weeks of conscription between January 2010 and July 2021, we are investigating whether the body mass index (BMI) of the group of young Swiss men after the two shutdown periods in Spring and Fall 2020 differs from the group of conscripts examined before the shutdowns. To our knowledge, this is also the first of such study internationally based on measured routine weight monitoring data. Comparable data from other countries could follow our results. 


\section{Methods}

\section{Swiss Conscription}

Conscription for the Swiss Armed Forces examines each year approximately 95\% of a birth cohort of men with Swiss citizenship one time at age between 18 and 22 years.(11) A detailed description of the conscription process can be found elsewhere.(11) Together with other examinations, the medical examination during conscription serves to check the state of health and thus the physical fitness for military service of the individual conscript. These examinations enable the health status of young men to be monitored with unique broad coverage. The six centres where conscription is conducted based on identical protocols are Payerne, Mels, Monteceneri, Rüti, Sumiswald, and Aarau. All measurements are performed by trained army medical personnel or specifically trained soldiers serving in the conscription process. In addition to health data, current occupation and place of residence were recorded.

In the summer of 2018, the Swiss Armed Forces introduced a more flexible conscription system into the armed forces, in the course of which young men themselves determine the timing of their conscription based on the desired date of basic training occurring approximately 12 months later (we control our analyses for a change in this system change).

\section{Variables}

The delivered anonymized data included the age groups of the conscripts (18.00-18.99 years, 19.00-19.99 years, 20.00-20.99 years, 21.00-21.99 years, and older than 22.00 years). To measure body height (cm) and weight $(\mathrm{kg})$ in underwear and without shoes, a stadiometer and a regular calibrated scale were used. Body mass index (BMI) (weight $[\mathrm{kg}] /$ height $\left[\mathrm{m}^{2}\right]$ ) was calculated.

Self-reported occupation of the conscripts is the only variable that indicates the socioeconomic background at the individual level. Following established procedures,(12) the occupations of conscripts were assigned to the Socio-Economic Index of Occupational Status (ISEI-08).(13) The ISEI allows the comparison of occupations according to their socioeconomic status. The ISEI-08 distribution of the occupations was divided into three tertile groups (low, medium, high). Pupils and students together form a separate group, as do those conscripts with no or insufficient information about their occupation. The regional attributes (language region (3), larger 
regions (Grossregionen, 7), MS region (microregional intermediate level for scientific and regional policy purposes, 106), urbanity) were linked to the data via residential postal code and place of residence before full anonymization and data delivery to the study team. The average Swiss neighbourhood index (Swiss-SEP 2.0) was also linked at the municipality level (14) Swiss-SEP 2.0 indicates the average socioeconomic situation in a postal code and was developed and made available by the Institute for Social and Preventive Medicine at the University of Bern. For this study, the Swiss-SEP of municipalities was divided into quintiles.

In the course of the COVID-19 pandemic and following national public health interventions, recruitment was suspended in Spring and Autumn 2020. During the first shutdown, conscription was stopped for a total of 13 weeks from calendar week 10 (Monday, March 09, 2020) to calendar week 20 (Friday, May 15, 2020). There was a second interruption of the conscription process for three weeks from calendar week 44 (Monday, October 26, 2020) until calendar week 46 (Friday, November 13, 2020). In both cases, we describe postshutdown effects as two 15 -week stretches, starting in the week of resumption after the shutdowns. This time window was determined before the data analysis. On the one hand, these almost 4 months are short enough to track BMI changes at the level of groups, on the other hand, this window is also long enough to allow for a sufficiently large sample size as well as not only describe short-term rather random fluctuations.

\section{Ethics}

The fully anonymized dataset for this retrospective cross-sectional monitoring study was provided by the Armed Forces Medical Services (AFMS) on a contractual basis. The data were exported by the AFMS from the "Medizinisches Informationssystem der Armee" (MEDISA) and were made completely anonymous before being handed over to the study team. As described earlier (11), Swiss conscription is mandatory and the anthropometric measurements used in this study are non-clinical, governmental data. According to Swiss federal law ("Bundesgesetz über die militärischen Informationssysteme” MIG, BG 510.91, Art. 2, 9, 24-29), the Swiss Army is authorized to make the data accessible for academic research in anonymous form. When dealing with fully anonymized and non-clinical data, no additional ethical approval is needed for analyses based on such governmental data (Swiss data privacy act, SR 235.1; 19.6.1992).

\section{Statistical analysis}


Our analysis is based on a model of the GAM (generalized additive model $(15,16)$ ) class, using penalized splines for smooth terms to achieve sufficient flexibility and t-distribution (with degree of freedom estimated from data) for the error term to achieve robustness to occasional data coding errors. The structure of the model for BMI of the $i$-th individual of residence municipality $s$ at time $t$ (coded in weeks since the beginning of the study) is correcting for spatial, temporal and individual heterogeneity nuisance effects to extract post-shutdown effects as follows:

$B M I_{\text {ist }}$

$=\beta_{0}+\beta_{\text {urban }} . I$ (municipality $s$ is of urban type $)+\sum_{k} \alpha_{k} . I$ (individual $i$ is of age class $k$ )

$+\sum_{l} \gamma_{l} . I$ (individual $i$ is of ISEI group $l$ )

$+\beta_{\text {scheme. }} I$ (time $t$ is before the change in conscription scheme $)+s_{\text {year }}($ year of $t)$

$+s_{\text {seasonality }}($ week of $t)+s_{S E P}($ mean SEP value of municipality $s)$

$+s_{\text {region }}($ MS region of municipality $s$ )

$+s_{\text {postshutdown } 1}$ (week after end of first shutdown). $I(t$ is within 15 weeks after the end of fist shutdown)

$+s_{\text {postshutdown } 2}$ (week after end of second shutdown). $I(t$ is within 15 weeks after the end of second shutdown)

$+\varepsilon_{i s t}$

where

- $I($.$) is an indicator function (assumes a value of 1$ if its argument is true and a value of 0 otherwise)

- $\beta_{0}, \beta_{\text {urban }}, \beta_{\text {scheme }}$ are unknown coefficients

- $\quad \alpha_{k}$ 's and $\gamma_{l}$ 's are unknown coefficients corresponding to the ANOVA-like submodels in age and ISEI factors (with usual baseline identifiability restrictions)

- $s_{y e a r}, s_{S E P}$ are smooth terms implemented as cubic splines

- $s_{\text {seasonality }}$ has to satisfy (as any proper seasonal profile) periodic conditions so that it is implemented as a cyclic cubic spline

- $s_{\text {region }}$ is the spatial random effect modelled as GMRF (Gaussian Markov random field) 
- $\varepsilon_{i s t} \sim t\left(0, v, \sigma^{2}\right)$ is a t-distributed error term with scale and degrees of freedom parameters estimated from the data. Use of t-distributed error term with rather low degrees of freedom (estimated from data), instead of traditional Gaussian errors makes the model highly robust - resistant to potential outliers that might occur in big-data that we model.

- $s_{\text {postlockdown } 1}$ and $s_{\text {postlockdown } 2}$ are smooth effects of main interest. They are implemented as cubic splines with the restriction $s_{\text {postlockdown } 1}(0)=s_{\text {postlockdown } 2}(0)=0$

All unknown coefficients and smooth terms are estimated from data via optimization of penalized likelihood, with penalty coefficients estimated by REML. The calculations were performed in R (17) with the help of the mgcv library (15). It is important to note that our GAM-based, semiparametric approach is a substantial generalization as compared to the linear or other pre-defined functional shape analysis promoted in traditional approaches like e.g., interrupted time series regression (18). In fact, the approach we use allows for non-linear and non-constant effects, as salient effects are important in our analysis of post-shutdown transient effects of a priori unknown functional shape.

As a sensitivity check, we employed also an ordinal regression model with WHO BMI categories and similar structure of the explanatory variables in order to check the results in an alternative and even more robust (or outlier-resistant) way.

\section{Results}

The dataset used for this analysis included 373,016 conscripts examined between January 1, 2010, and July 31, 2021. There were no extreme and unreliable values in height or weight we had to exclude. The relative frequency of age groups, greater regions, and occupational groups did not markedly change over time (Table 1). In 2020 (the most recent full calendar year included in the dataset), the mean BMI was $22.30 \mathrm{~kg} / \mathrm{m}^{2}$ (SD 4.0), $19.6 \%$ of the conscripts were overweight, and $5.9 \%$ were obese. For the first six months of 2021, for which data were already available, the BMI of young men did not change significantly compared to 2020 . Overall, the mean BMI and the prevalence of overweight and obesity stabilized at a high level since 2010 (Table 1). 
Our best fitting GAM model (7.7\% deviance explained) included two separate postshutdown effects of 15 weeks each, starting at the end of the shutdowns, and included smoothed terms (full model results see appendix Chapter 1. Figure 1 shows that after the first shutdown in Spring 2020, the BMI of the conscripts entering conscription 15 weeks after the temporary suspension of conscription during the shutdown was not significantly different from that of the conscripts before the shutdown (Figure 1A). However, there was a significant effect $(\mathrm{p}=0.011)$ after the second, shorter shutdown in Fall 2020 (Figure 1B), with a slight increase in BMI in the first 2 weeks, followed by a decrease in BMI until week 15 of the post-shutdown effect. In both cases in Figure 1, the confidence intervals are relatively wide, indicating a rather weaker effect.

Our main model also revealed significant differences in BMI for the other included covariables (detailed results can be found in the Supplementary Appendix): Compared to the reference group of the 19-year-old conscripts, older conscripts had a significantly higher BMI, and conscripts with a higher ISEI group and students had a significantly lower BMI. We also found that conscripts living in urban and lower Swiss-SEP areas of Switzerland had a higher BMI (the smoothed effect was almost linear, see Appendix 1.1, Figure B). There was also a significant spatial effect across the 106 MS regions in Switzerland, with the northwestern part of Switzerland having higher BMI values. We also found weak but significant effects for seasonality (lowest BMI values in the summer months) and across years, when the average BMI of Swiss conscripts slightly decreased since 2010 .

The same conclusion (only weak effects of shutdown periods on BMI) can be drawn when the model was stratified by larger regions or when we used the heteroskedastic GAMLSS model as an alternative (taking into account the nature of the BMI distribution). The conclusion also did not change when using ordinal regression models to assess WHO categories for BMI.

\section{Discussion}

In this paper, we analysed whether shutdowns during the COVID-19 pandemic affected the measured body weight of young men in Switzerland. We showed that the BMI of conscripts who were examined in the 15 weeks after the two shutdowns in Spring and Autumn 2020 was not or only weakly different from baseline. Sensitivity analyses showed that this conclusion also holds if assessing the BMI distribution or the prevalence 
of overweight and/or obesity. The models further showed strong effects of individual and area-based SEP as well as age on BMI.

Because the number of similar studies based on measured monitoring data is still very small, contextualization with the literature is difficult at the moment. The comparison with the many survey studies based on selfreported before-after weight data is challenging in many aspects. Many of these survey studies show weight gain during the pandemic, especially for women, previously overweight people, and older people.(19) This was also recently shown by the only Swiss study on this topic.(10) The reasons for the young men in our data not gaining weight may be multifactorial. One possible explanation could be that the shutdowns in Switzerland were relatively unrestrictive. Despite the recommendation to stay at home, the Swiss population was allowed to go outside at any time. Even with visible shutdown effects in national mobility tracking,(3) the more local movement patterns of young people might not have been restricted. Another possibility is that changes in the balance between diet and exercise level over several weeks are less likely to translate into additional body weight in younger people than in older people. One possible explanation could be that lipid turnover in fat tissue decreases during ageing.(20) In future events such as shutdowns, health monitoring of young men should include not only anthropometric parameters but also body composition and metabolic parameters, which are particularly relevant in terms of later health risk.

Our study had several limitations. First, we only considered young Swiss men in our study. Women were not considered, even though they are particularly at risk of gaining weight according to a French study.(21) A second limitation is that we only have cross-sectional data. To make a statement about the weight profile of an individual, it would be necessary to collect longitudinal, individual measurements before and after the pandemic. At the moment, such data only exist in the form of self-reported weight information. Furthermore, we have no information on how the lifestyle of young men has changed during shutdowns, especially regarding diet, physical activity, psychological condition and well-being. However, this information would be crucial to put the observed effect in a broader context. Last but not least, it is a limitation of BMI that it only reflects the relation between weight and height and cannot say anything about body composition. Therefore, changes in body composition and/or metabolic parameters could also have occurred in young men during and after the shutdowns, which, however, were not reflected in additional body weight. 
Prevention is the most effective therapy. $(20,22)$ It is important to understand how the behaviour and health of a population adapt and change in response to a crisis on a larger scale. Surveillance and monitoring of the population and public health are the basic prerequisites for possible targeted intervention and are therefore of paramount importance. This especially applies to young men because being obese in adolescence increases the risk of being obese as an adult, and the disease risks associated with excess weight in men in particular increase with age.(20) Certainly, it would be wrong to say that no prevention against weight gain is needed in the event of another shutdown event among young men based on our study results. For successful prevention, not only should risk factors be addressed, but underlying risk-reducing resources should be identified and strengthened. For this purpose, our study population could also be helpful for further studies for other population groups (women, children, elderly people), not only in Switzerland but also in other countries. 


\section{Author statements}

\section{Funding sources}

The authors thank the Swiss Armed Forces (Grantee Kaspar Staub), Mäxi Foundation (Grantee Frank Rühli), and the long-term strategic development financing of the Institute of Computer Science (Czech Republic RVO 67985807, Marek Brabec) for financial support.

\section{Acknowledgements}

The authors are especially thankful to Major General Andreas Stettbacher, Surgeon General of the Swiss Armed Forces, for providing the anonymized conscription data, as well as Tiziano Angelelli and Franz Frey for their research support.

\section{Conflict of Interest Statement}

The authors do not declare a conflict of interest.

\section{Data Availability Statement}

This article used fully anonymized individual data as provided by the Swiss Armed Forces upon signing a data contract. By this contract, the authors of the article are not authorized to make individual datasets publicly available. However, other researchers may request the very same data set from the Swiss Armed Forces via submission of a study protocol. The R-code for the analysis of the datasets can be obtained from the corresponding author. 
Key-points

- We were able to show, based on measured monitoring data, that the weight of young men was only slightly influenced by environmental changes such as mobility restrictions or home office due to the corona-related shutdowns.

- Over the last 10 years, the BMI of young Swiss people has stabilised at a high level, with those living in urban areas having a significantly higher BMI than those living in rural areas.

- In the case of a negative study outcome regarding BMI increase due to shutdowns, our study population can be used to identify and strengthen underlying risk-reducing resources that could be useful for other more vulnerable populations. 


\section{References}

1. Bundesamt für Gesundheit BAG. Massnahmen und Verordnungen.

2. Effects of COVID-19 Home Confinement on Eating Behaviour and Physical Activity: .

3. Amt AS, Covid- SN, Force ST, Konjunkturforschungsstelle KOF. Mobilitäts-Monitoring COVID-19. 2021.

4. Huber BC, Steffen J, Schlichtiger J, Brunner S. Altered nutrition behavior during COVID-19 pandemic lockdown in young adults. Eur J Nutr. 2021;60(5):2593-602.

5. Deschasaux-Tanguy M, Druesne-Pecollo N, Esseddik Y, De Edelenyi FS, Allès B, Andreeva VA, et al. Diet and physical activity during the coronavirus disease 2019 (COVID-19) lockdown (MarchMay 2020): Results from the French NutriNet-Santé cohort study. Am J Clin Nutr. 2021;113(4):92438.

6. López-Moreno M, López MTI, Miguel M, Garcés-Rimón M. Physical and psychological effects related to food habits and lifestyle changes derived from covid-19 home confinement in the spanish population. Nutrients. 2020;12(11):1-17.

7. Marty L, de Lauzon-Guillain B, Labesse M, Nicklaus S. Food choice motives and the nutritional quality of diet during the COVID-19 lockdown in France. Appetite. 2021 Feb;157.

8. Bakaloudi DR, Barazzoni R, Bischoff SC, Breda J, Wickramasinghe K, Chourdakis M. Impact of the first COVID-19 lockdown on body weight: A combined systematic review and a meta-analysis. Clin Nutr. 2021;(xxxx).

9. Khan MAB, Menon P, Govender R, Samra A, Nauman J, Ostlundh L, et al. Systematic review of the effects of pandemic confinements on body weight and their determinants. Br J Nutr. 2021;(7).

10. Rudolph T, Eggenschwiler M, Kralle N. Food Consumption 2021. 2021;(August).

11. Panczak R, Zwahlen M, Woitek U, Rühli FJ, Staub K. Socioeconomic, temporal and regional variation in body mass index among 188,537 Swiss male conscripts born between 1986 and 1992. Schooling CM, editor. PLoS One [Internet]. 2014 May 12 [cited 2014 May 12];9(5):e96721. Available from: http://dx.plos.org/10.1371/journal.pone.0096721 
12. Floris J, Koepke N, Bender N, Rühli PF, Staub K. Der Body-Mass-Index der Schweizer Stellungspflichtigen 2015. Bericht zuhanden des Bundesamtes für Gesundh (BAG-Vertragsnummer. $2016 ; 1-35$.

13. Ganzeboom HBG. A New International Socio-Economic Index (ISEI) of Occupational Status for the International Standard Classification of Occupation 2008 (ISCO 08). Constructed with Data from the ISSP 2002-2007. Paper presented at Annual Conference of International Social . 2010;

14. Panczak R, Galobardes B, Voorpostel M, Spoerri A, Zwahlen M, Egger M. A Swiss neighbourhood index of socioeconomic position: development and association with mortality.

15. Wood SN. Generalized additive models: An introduction with R. 2nd ed. Generalized Additive Models: An Introduction with R. Boca Raton: Chapman and Hall/CRC; 2017. 1-496 p.

16. Hastie TJ, Tibshirani RJ. Generalized additive models. 1st ed. Generalized Additive Models. Boca Raton: Routledge; 1990. 1-352 p.

17. R-Foundation. The R Project for Statistical Computing.

18. Lopez Bernal J, Cummins S, Gasparrini A. Interrupted time series regression for the evaluation of public health interventions: a tutorial. Int J Epidemiol. 2016 Jun 9;dyw098.

19. Di Renzo L, Gualtieri P, Pivari F, Soldati L, Attinà A, Cinelli G, et al. Eating habits and lifestyle changes during COVID-19 lockdown: An Italian survey. J Transl Med. 2020;18(1):1-15.

20. Hutton G, Water S and HT, World Health Organization. Considerations in evaluating the cost-effectiveness of environmental health interventions. Geneva; 2000.

21. Deschasaux-Tanguy M, Druesne-Pecollo N, Esseddik Y, de Edelenyi FS, Allès B, Andreeva VA, et al. Diet and physical activity during the COVID-19 lockdown period (March-May 2020): Results from the French NutriNet-Santé cohort study. The American Journal of Clinical Nutrition. 2020. p. 15.

22. van der Vliet N, Suijkerbuijk AWM, de Blaeij AT, Ardine de Wit G, van Gils PF, Staatsen BAM, et al. Ranking preventive interventions from different policy domains: What are the most cost-effective ways to improve public health? Int J Environ Res Public Health. 2020;17(6). 
Table 1: Data overview

\begin{tabular}{|c|c|c|c|c|c|c|c|c|c|c|c|c|c|c|c|c|c|c|c|c|c|c|c|c|}
\hline $\begin{array}{l}\text { Year } \\
\text { Conscripts }\end{array}$ & $\begin{array}{r}2010 \\
n\end{array}$ & & $\begin{array}{r}2011 \\
n\end{array}$ & & 2012 & & 2013 & & 2014 & & 2015 & & 2016 & & 2017 & & 2018 & & 2019 & & 2020 & & 2021 & \\
\hline Total & 36792 & & 37251 & & 36272 & & 35673 & & 34914 & & 34724 & & 33785 & & 32201 & & 38188 & & 24517 & & 18869 & & 9830 & \\
\hline Agegroups & $\mathrm{n}$ & $\%$ & $\mathbf{n}$ & $\%$ & $\mathrm{n}$ & $\%$ & $\mathrm{n}$ & $\%$ & $\mathrm{n}$ & $\%$ & $\mathrm{n}$ & $\%$ & $\mathrm{n}$ & $\%$ & $\mathrm{n}$ & $\%$ & $\mathrm{n}$ & $\%$ & $\mathrm{n}$ & $\%$ & $\mathrm{n}$ & $\%$ & $\mathrm{n}$ & $\%$ \\
\hline$<19.00$ & 8534 & 23.2 & 8849 & 23.8 & 8890 & 24.5 & 8902 & 25.0 & \begin{tabular}{|l|l|}
10819 \\
\end{tabular} & 31.0 & 10832 & 31.2 & \begin{tabular}{|l|l|}
10818 \\
\end{tabular} & 32.0 & \begin{tabular}{|l|l|}
11514 \\
\end{tabular} & 35.8 & \begin{tabular}{|l|l}
13327 \\
\end{tabular} & 34.9 & 8831 & 36.0 & 4467 & 23.7 & 2306 & 23.5 \\
\hline $19.00-19.99$ & 16751 & 45.5 & 17234 & 46.3 & 16702 & 46.0 & $\mid 16413$ & 46.0 & $\mid 15702$ & 45.0 & $\mid 15726$ & 45.3 & 15479 & 45.8 & 13785 & 42.8 & 15666 & 41.0 & 10208 & 41.6 & 8048 & 42.7 & 3744 & 38.1 \\
\hline $20.00-20.99$ & 8489 & 23.1 & 8298 & 22.3 & \begin{tabular}{|l}
7784 \\
\end{tabular} & 21.5 & 7575 & 21.2 & 6170 & 17.7 & 6058 & 17.4 & 5419 & 16.0 & 5212 & 16.2 & 6913 & 18.1 & 4309 & 17.6 & 4609 & 24.4 & 2614 & 26.6 \\
\hline $21.00-21.99$ & 3018 & 8.2 & 2870 & 7.7 & 2896 & 8.0 & 2783 & 7.8 & 2223 & 6.4 & 2108 & 6.1 & 2069 & 6.1 & 1690 & 5.2 & 2282 & 6.0 & 1169 & 4.8 & 1745 & 9.2 & 1166 & 11.9 \\
\hline Grossregionen & $\mathrm{n}$ & $\%$ & $\mathrm{n}$ & $\%$ & $\mathbf{n}$ & $\%$ & $\mathbf{n}$ & $\%$ & $\mathbf{n}$ & $\%$ & $\mathbf{n}$ & $\%$ & $\mathbf{n}$ & $\%$ & $\mathrm{n}$ & $\%$ & $\mathrm{n}$ & $\%$ & $\mathrm{n}$ & $\%$ & $\mathbf{n}$ & $\%$ & $\mathrm{n}$ & $\%$ \\
\hline Central Switzerland & 4103 & 11.2 & 4038 & 10.8 & 4072 & 11.2 & 3901 & 10.9 & 3844 & 11.0 & 3792 & 10.9 & 3740 & 11.1 & 3625 & 11.3 & 2917 & 7.6 & 2735 & 11.2 & 2311 & 12.2 & 1064 & 10.8 \\
\hline Eastern Switzerland & 6128 & 16.7 & 6218 & 16.7 & 5859 & 16.2 & 5293 & 14.8 & 5293 & 15.2 & 5598 & 16.1 & 5176 & 15.3 & 5073 & 15.8 & 4519 & 11.8 & 3926 & 16.0 & 2963 & 15.7 & 1311 & 13.3 \\
\hline Espace Mittelland & 9191 & 25.0 & 8867 & 23.8 & 8282 & 22.8 & 8767 & 24.6 & 8524 & 24.4 & 8131 & 23.4 & 7388 & 21.9 & 7269 & 22.6 & 12515 & 32.8 & 6122 & 25.0 & 4550 & 24.1 & 2441 & 24.8 \\
\hline Lake Geneva Region & 5362 & 14.6 & 5539 & 14.9 & 6116 & 16.9 & 6359 & 17.8 & 5986 & 17.1 & 5805 & 16.7 & 5795 & 17.2 & 5258 & 16.3 & 8839 & 23.1 & 3393 & 13.8 & 2602 & 13.8 & 1354 & 13.8 \\
\hline Northwestern Switzerland & 4971 & 13.5 & 4972 & 13.3 & 4939 & 13.6 & 4884 & 13.7 & 4936 & 14.1 & 4756 & 13.7 & 4420 & 13.1 & 4463 & 13.9 & 3240 & 8.5 & 3284 & 13.4 & 2480 & 13.1 & 1410 & 14.3 \\
\hline Ticino & 1421 & 3.9 & 1372 & 3.7 & 1377 & 3.8 & 1349 & 3.8 & 1308 & 3.7 & 1323 & 3.8 & 1402 & 4.1 & 1325 & 4.1 & 1344 & 3.5 & 1045 & 4.3 & 813 & 4.3 & 453 & 4.6 \\
\hline Zurich & 5615 & 15.3 & 6244 & 16.8 & 5627 & 15.5 & 5112 & 14.3 & 5018 & 14.4 & 5304 & 15.3 & 5863 & 17.4 & 5187 & 16.1 & 4811 & 12.6 & 4010 & 16.4 & 3148 & 16.7 & 1796 & 18.3 \\
\hline ISEI-Groups & $\mathbf{n}$ & $\%$ & $\mathbf{n}$ & $\%$ & $\mathbf{n}$ & $\%$ & $\mathbf{n}$ & $\%$ & $\mathbf{n}$ & $\%$ & $\mathbf{n}$ & $\%$ & $\mathrm{n}$ & $\%$ & $\mathrm{n}$ & $\%$ & $\mathrm{n}$ & $\%$ & $\mathbf{n}$ & $\%$ & $\mathbf{n}$ & $\%$ & $\mathbf{n}$ & $\%$ \\
\hline Tertile 1 (low) & 8428 & 22.9 & 8317 & 22.3 & 7504 & 20.7 & 7321 & 20.5 & 7119 & 20.4 & 6952 & 20.0 & 6591 & 19.5 & 6141 & 19.1 & 6719 & 17.6 & 4942 & 20.2 & 3835 & 20.3 & 1681 & 17.1 \\
\hline Tertile 2 (medium) & 7998 & 21.7 & 7916 & 21.3 & 7904 & 21.8 & 7745 & 21.7 & 6919 & 19.8 & 6758 & 19.5 & 8963 & 26.5 & 8428 & 26.2 & 9058 & 23.7 & 6439 & 26.3 & 5235 & 27.7 & 2366 & 24.1 \\
\hline Tertile 3 (high) & 7321 & 19.9 & 7658 & 20.6 & 7584 & 20.9 & 7516 & 21.1 & 7341 & 21.0 & 7365 & 21.2 & 5176 & 15.3 & 4905 & 15.2 & 5487 & 14.4 & 4026 & 16.4 & 3260 & 17.3 & 1503 & 15.3 \\
\hline Students & 9964 & 27.1 & 9740 & 26.1 & 9626 & 26.5 & 9195 & 25.8 & 9508 & 27.2 & 9830 & 28.3 & 8704 & 25.8 & 7438 & 23.1 & 8822 & 23.1 & 5590 & 22.8 & 3565 & 18.9 & 2429 & 24.7 \\
\hline Imprecise & 3080 & 8.4 & 3620 & 9.7 & 3654 & 10.1 & 3896 & 10.9 & 4027 & 11.5 & 3819 & 11.0 & 4351 & 12.9 & 5289 & 16.4 & 8099 & 21.2 & 3520 & 14.4 & 2974 & 15.8 & 1851 & 18.8 \\
\hline Mean values & $\mathrm{m}$ & so & $\mathrm{m}$ & $s d$ & $\mathbf{m}$ & sd & $\mathbf{m}$ & sd & $\mathbf{m}$ & $s d$ & $\mathrm{~m}$ & $s d$ & m & $s d$ & $\mathbf{m}$ & $s d$ & $\mathbf{m}$ & $s d$ & m & $s d$ & $\mathrm{~m}$ & sd & $\mathbf{m}$ & $s d$ \\
\hline Height [cm] & 178.2 & 6.5 & 178.2 & 6.5 & 178.3 & 6.6 & 178.2 & 6.6 & 178.3 & 6.6 & 178.4 & 6.6 & 178.4 & 6.6 & 178.5 & 6.6 & 178.5 & 6.6 & 178.8 & 6.6 & 178.7 & 6.6 & $\mid 178.7$ & 6.6 \\
\hline Weight [kg] & 74.5 & 13.1 & 74.5 & 13.0 & 74.5 & 13.1 & 74.4 & 13.2 & 74.3 & 13.2 & 74.2 & 13.3 & 74.4 & 13.5 & 74.3 & 13.9 & 74.3 & 13.8 & 74.4 & 13.4 & 74.6 & 13.7 & 74.5 & 14.0 \\
\hline BMI $\left[\mathrm{kg} / \mathrm{m}^{2}\right]$ & 23.4 & 3.8 & 23.4 & 3.8 & 23.4 & 3.8 & 23.4 & 3.8 & 23.3 & 3.8 & 23.3 & 3.9 & 23.4 & 3.9 & 23.3 & 4.0 & 23.3 & 4.0 & 23.3 & 3.9 & 23.3 & 4.0 & 23.3 & 4.1 \\
\hline BMI $[\mathrm{kg} / \mathrm{m}$ & & & & & & & & & & & & & & & & & & & & & & & & \\
\hline (WHO categories) & $\mathrm{n}$ & $\%$ & $\mathrm{n}$ & $\%$ & $\mathbf{n}$ & $\%$ & $\mathbf{n}$ & $\%$ & $\mathbf{n}$ & $\%$ & $\mathbf{n}$ & $\%$ & $\mathbf{n}$ & $\%$ & $\mathbf{n}$ & $\%$ & $\mathbf{n}$ & $\%$ & $\mathbf{n}$ & $\%$ & $\mathbf{n}$ & $\%$ & $n$ & $\%$ \\
\hline$<18.5$ & 1290 & 3.5 & 1374 & 3.7 & 1402 & 3.9 & 1421 & 4.0 & 1535 & 4.4 & 1640 & 4.7 & 1655 & 4.9 & 1738 & 5.4 & 2060 & 3.5 & 1328 & 3.5 & 1019 & 3.5 & 575 & 3.5 \\
\hline $18.5-24.9$ & 26125 & 71.0 & 26409 & 70.9 & 25671 & 70.8 & 25300 & 70.9 & 24805 & 71.0 & 24531 & 70.6 & 23438 & 69.4 & 22313 & 69.3 & 25018 & 71.0 & 16934 & 71.0 & 12944 & 71.0 & 6718 & 71.0 \\
\hline $25.0-29.9$ & 7220 & 19.6 & 7257 & 19.5 & \begin{tabular}{|l|l}
7077 \\
\end{tabular} & 19.5 & 6835 & 19.2 & 6529 & 18.7 & 6445 & 18.6 & 6584 & 19.5 & 6039 & 18.8 & \begin{tabular}{|l}
6687 \\
\end{tabular} & 19.6 & 4658 & 19.6 & 3579 & 19.6 & 1828 & 19.6 \\
\hline$\geq 30.0$ & 2157 & 5.9 & 2211 & 5.9 & 2122 & 5.9 & 2117 & 5.9 & 2045 & 5.9 & 2108 & 6.1 & 2106 & 6.2 & 2111 & 6.6 & 2324 & 5.9 & 1524 & 5.9 & 1255 & 5.9 & 652 & 5.9 \\
\hline
\end{tabular}




\section{Figure legend}

Figure 1: Population-level changes in young men's BMI after the first (A, Spring 2020) and second (B, autumn 2020) shutdown ( $s_{\text {postshutdown } 1}$ and $s_{\text {postshutdown } 2}$ model terms). The y-axis indicates the effect (partial changes in BMI ). The $\mathrm{x}$-axis indicates the time in weeks after the end of the shutdown. Upper and lower limits of the grey area correspond to the (pointwise constructed) 95\% asymptotic intervals.

A

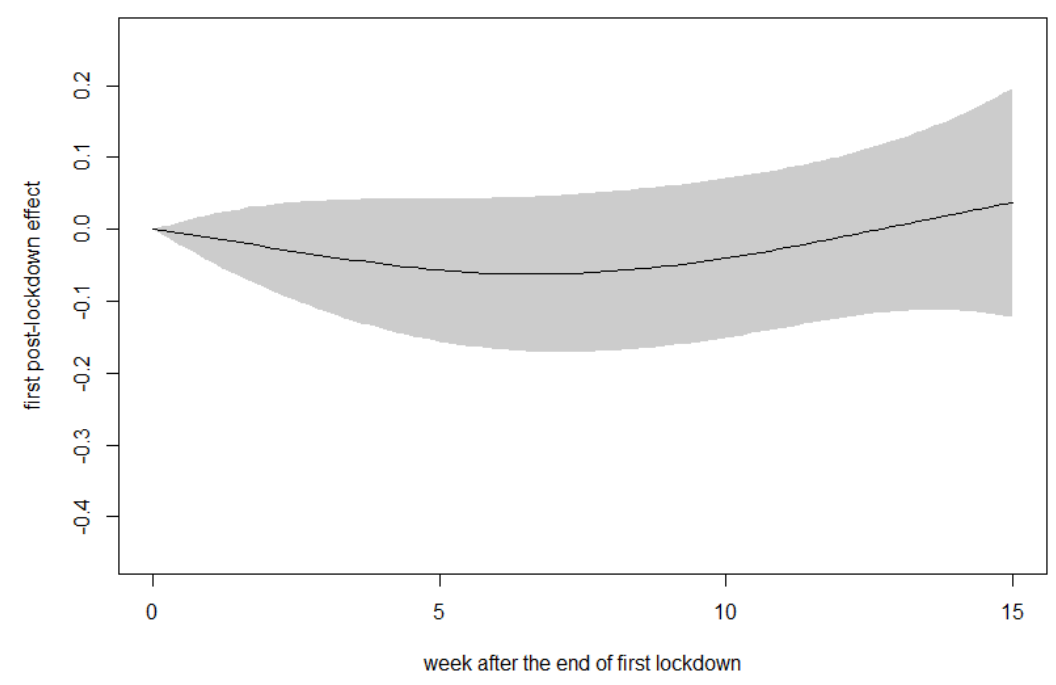

B

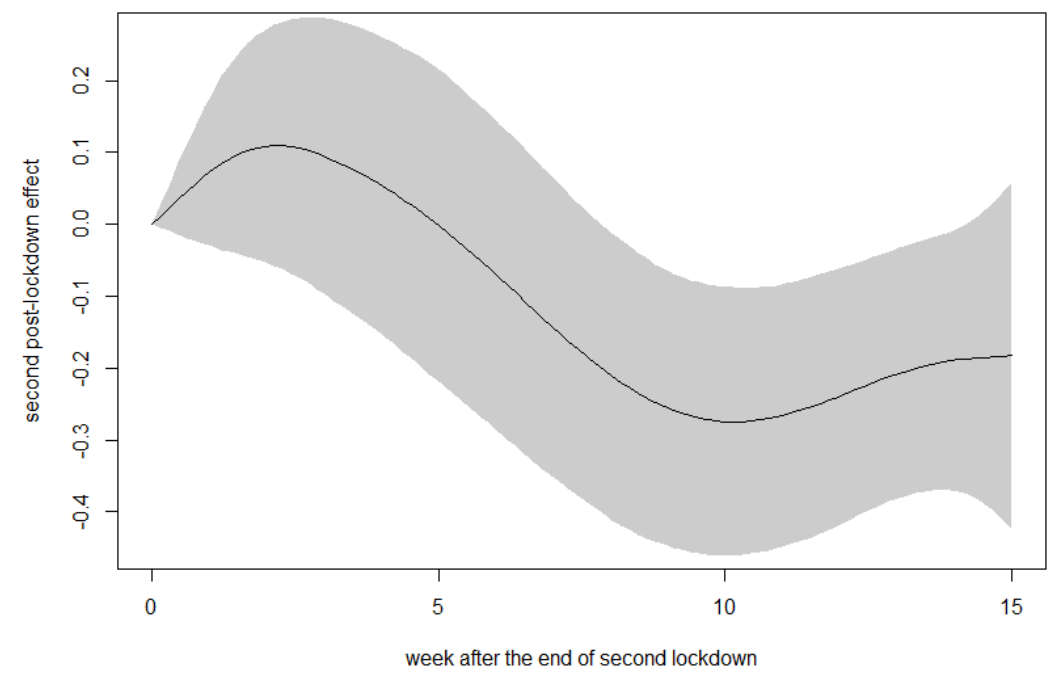




\section{Appendix Material to:}

Body mass index in young men in Switzerland after the national shutdowns during the COVID-19 pandemic - Results from a cross-sectional monitoring study at population level since 2010.

Samuel Meili, Marek Brabec, Frank Rühli, Thomas W. Buehrer, Nejla Gültekin, Zeno Stanga, Nicole Bender, Kaspar Staub, Emilie Reber

\section{Outline:}

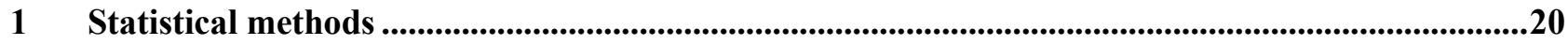

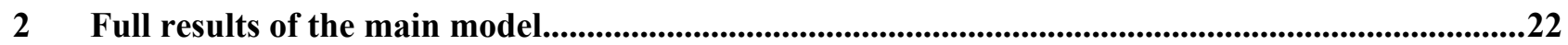

2.1 Visualization of the smoothed terms and MS region effect on BMI.............................................24

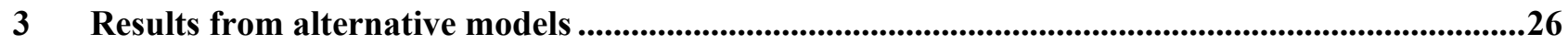

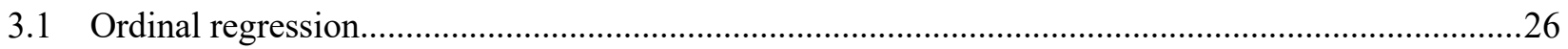

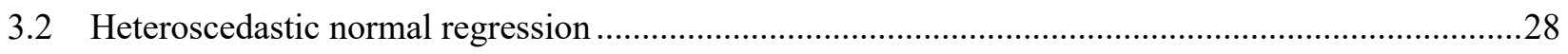




\section{Statistical methods}

Our analysis is based on a model of the (slightly generalized) GAM (generalized additive model $(15,16)$ ) class, using penalized splines for smooth terms to achieve sufficient flexibility and t-distribution (with degree of freedom estimated from data) for the error term to achieve robustness to occasional data coding errors. The structure of the model for BMI of the $i$-th individual of residence municipality $s$ at time $t$ (coded in weeks since the beginning of the study) is correcting for spatial, temporal and individual heterogeneity nuisance effects to extract post-shutdown effects as follows:

$B M I_{i s t}$

$=\beta_{0}+\beta_{\text {urban }} . I$ (municipality $s$ is of urban type) $+\sum_{k} \alpha_{k} \cdot I$ (individual $i$ is of age class $k$ )

$+\sum_{l} \gamma_{l} . I$ (individual $i$ is of ISEI group $l$ )

$+\beta_{\text {scheme. }} I($ time $t$ is before the change in conscription scheme $)+s_{\text {year }}($ year of $t)$

$+s_{\text {seasonality }}($ week of $t)+s_{S E P}($ mean SEP value of municipality $s)$

$+s_{\text {region }}($ MS region of municipality $s$ )

$+s_{\text {postshutdown } 1}($ week after end of first shutdown). $I(t$ is within 15 weeks after the end of fist shutdown)

$+s_{\text {postshutdown } 2}$ (week after end of second shutdown). $I(t$ is within 15 weeks after the end of second shutdown)

$+\varepsilon_{i s t}$

where

- $I($.$) is an indicator function (assumes a value of 1$ if its argument is true and a value of 0 otherwise)

- $\beta_{0}, \beta_{\text {urban }}, \beta_{\text {scheme }}$ are unknown coefficients

- $\quad \alpha_{k}$ 's and $\gamma_{l}$ 's are unknown coefficients corresponding to the ANOVA-like submodels in age and ISEI factors (with usual baseline identifiability restrictions)

- $s_{y e a r}, s_{S E P}$ are smooth terms implemented as cubic splines 
- $s_{\text {seasonality }}$ has to satisfy (as any proper seasonal profile) periodic conditions so that it is implemented as a cyclic cubic spline

- $s_{\text {region }}$ is the spatial random effect modelled as GMRF (Gaussian Markov random field)

- $\varepsilon_{i s t} \sim t\left(0, v, \sigma^{2}\right)$ is a t-distributed error term with scale and degrees of freedom parameters estimated from the data.

- $s_{\text {postlockdown } 1}$ and $s_{\text {postlockdown } 2}$ are smooth effects of main interest. They are implemented as cubic splines with the restrictions postlockdown $1_{1}(0)=s_{\text {postlockdown } 2}(0)=0$

All unknown coefficients and smooth terms are estimated from data via optimization of penalized likelihood, with penalty coefficients estimated by REML. 


\section{Full results of the main model}

Fami1y: Scaled t(3.735,2.679)

Link function: identity

Formula:

$\mathrm{BMI} \sim \mathrm{s}($ Year, bs $=" \mathrm{cr} ")+$ furbanNon. Urban + fAgegroup + fscheme + fISEIGroups + s(Mean_SSEP, bs = "cr") + s(Week_Mon, bs $=" c c ")+s($ fMSRegion, bs $=$ "mrf", xt $=x$. MSRegion $)+$ $\mathrm{s}($ postlockdown 1 , by $=$ zero, bs $=" \mathrm{cr} ", \mathrm{pc}=0)+\mathrm{s}($ postlockdown 2 , by $=$ zero, bs $=$ "cr", pc $=0$ )

Parametric Terms:

$$
\text { df } \quad F \quad p \text {-value }
$$

furbanNon.urban $124.327 \quad 8.13 \mathrm{e}-07$

fagegroup $\quad 3756.106<2 \mathrm{e}-16$

$\begin{array}{llll}\text { fscheme } & 1 & 0.003 & 0.957\end{array}$

fISEIGroups $\quad 4434.268<2 \mathrm{e}-16$

Approximate significance of smooth terms:

$$
\text { edf Ref.df } F \text { p-value }
$$

s(Year) $\quad 5.1276 .22830 .740<2 \mathrm{e}-16$

S(Mean_SSEP) $\quad 3.9664 .913133 .142<2 \mathrm{e}-16$

s(Week_Mon) $\quad 6.093 \quad 8.000 \quad 9.554 \quad 2.51 \mathrm{e}-16$

s(fMSRegion) $91.078101 .50721 .928<2 \mathrm{e}-16$

$\begin{array}{lllll}\mathrm{s}(\text { postlockdown } 1) \text { : zero } & 1.750 & 2.136 & 0.744 & 0.4679\end{array}$

$\begin{array}{lllll}\text { s(postlockdown2) : zero } \quad 3.240 & 3.948 & 3.459 & 0.0108\end{array}$

$>\operatorname{summary}(\mathrm{a} . \mathrm{cs} .2)$

Fami1y: Scaled $\mathrm{t}(3.735,2.679)$

Link function: identity

Formu1a: 
$\mathrm{BMI} \sim \mathrm{s}($ Year, bs $=$ "cr") + furbanNon. Urban + fAgegroup +

fscheme + fISEIGroups + s(Mean_SSEP, bs = "cr") + s(Week_Mon, bs $=" c c ")+s($ fMSRegion, bs $=$ "mrf", xt $=x$. MSRegion $)+$ $\mathrm{s}($ postlockdown 1 , by $=$ zero, bs $=" \mathrm{cr} ", \mathrm{pc}=0)+\mathrm{s}($ post1ockdown 2 , by $=$ zero, bs $=$ "cr", $p c=0$ )

Parametric coefficients:

Estimate std. Error $t$ value $\operatorname{Pr}(>|t|)$

(Intercept)

furbanNon. Urbanurban

fagegroup20.00-20.99

fagegroup21.00-21.99

fschemeold fagegroup $<19.00$

22.914251

0.069570

0.318353

0.686759

$-0.291254$

0.001864

fISEIGroupswithout occupation -0.426096

fISEIGroupsTertile 1 (low)

0.109151

fISEIGroupsTertile 3 (high)

fISEIGroupsstudents
$-0.145160$

$-0.549195$
$0.030166759 .601<2 \mathrm{e}-16 * * *$

$0.014105 \quad 4.9328 .13 \mathrm{e}-07 * * *$

$0.01507021 .124<2 \mathrm{e}-16 * * *$

$0.02293529 .944<2 \mathrm{e}-16 * * *$

$0.013667-21.310<2 \mathrm{e}-16 * * *$

$\begin{array}{lll}0.034516 & 0.054 & 0.957\end{array}$

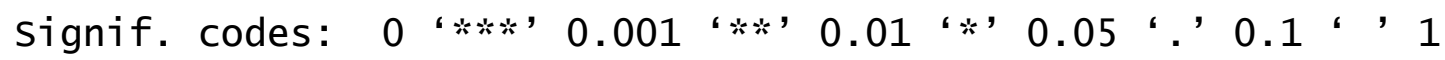

Approximate significance of smooth terms:

$\begin{array}{lrrrc} & \text { edf } & \text { Ref.df } & \text { F } & \text { p-value } \\ \text { s(Year) } & 5.127 & 6.228 & 30.740 & <2 \mathrm{e}-16 * * * \\ \text { s(Mean_SSEP) } & 3.966 & 4.913 & 133.142 & <2 \mathrm{e}-16 * * * \\ \text { s(Week_Mon) } & 6.093 & 8.000 & 9.554 & 2.51 \mathrm{e}-16 * * * \\ \text { s(fMSRegion) } & 91.078 & 101.507 & 21.928 & <2 \mathrm{e}-16 * * * \\ \text { s(postlockdown1):zero } & 1.750 & 2.136 & 0.744 & 0.4679 \\ \text { s(postlockdown2):zero } & 3.240 & 3.948 & 3.459 & 0.0108 *\end{array}$

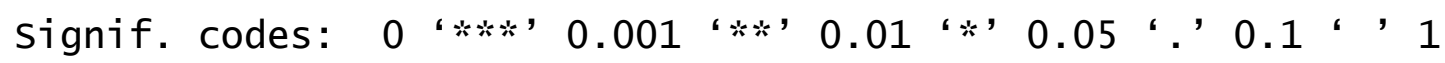

R-sq. $($ adj $)=0.0218$ Deviance explained $=7.74 \%$ 
fREML $=4.7316 \mathrm{e}+05$ scale est. $=1$

$\mathrm{n}=332320$

$>\operatorname{AIC}($ a.cs.2)

[1] 1785788

2.1 Visualization of the smoothed terms and MS region effect on BMI

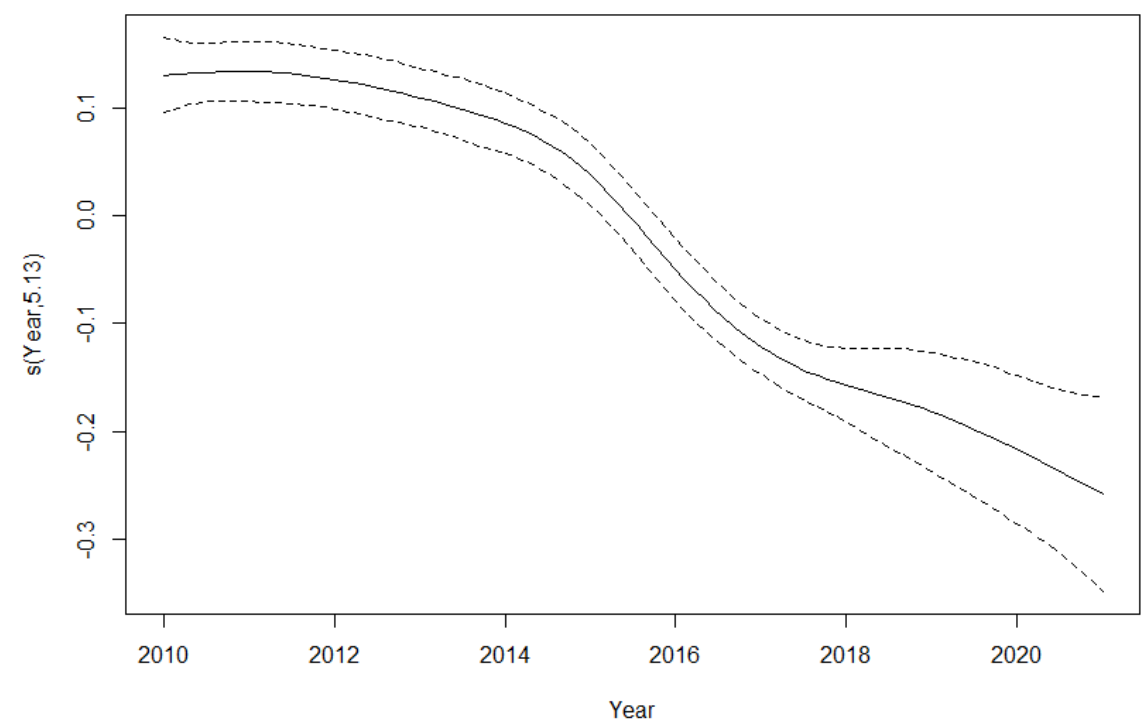

Figure A: Smoothed BMI effect across years

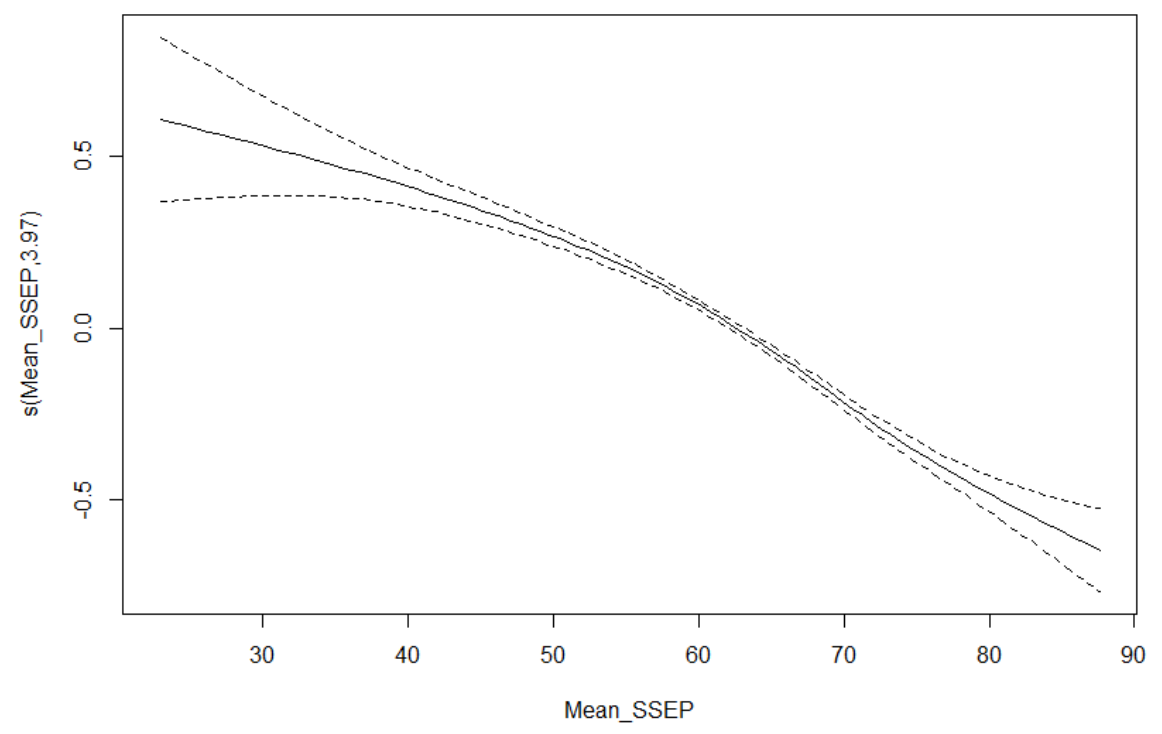

Figure B: Smoothed BMI effect across SSEP 


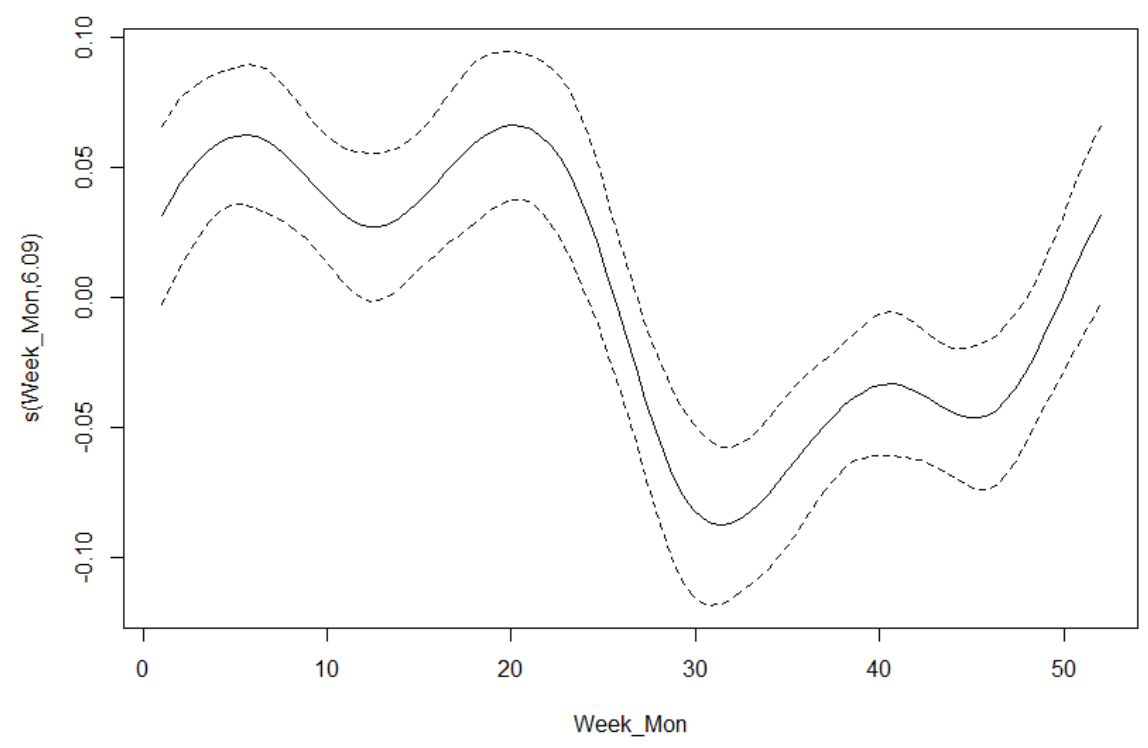

Figure C: Smoothed BMI effect across Weeks

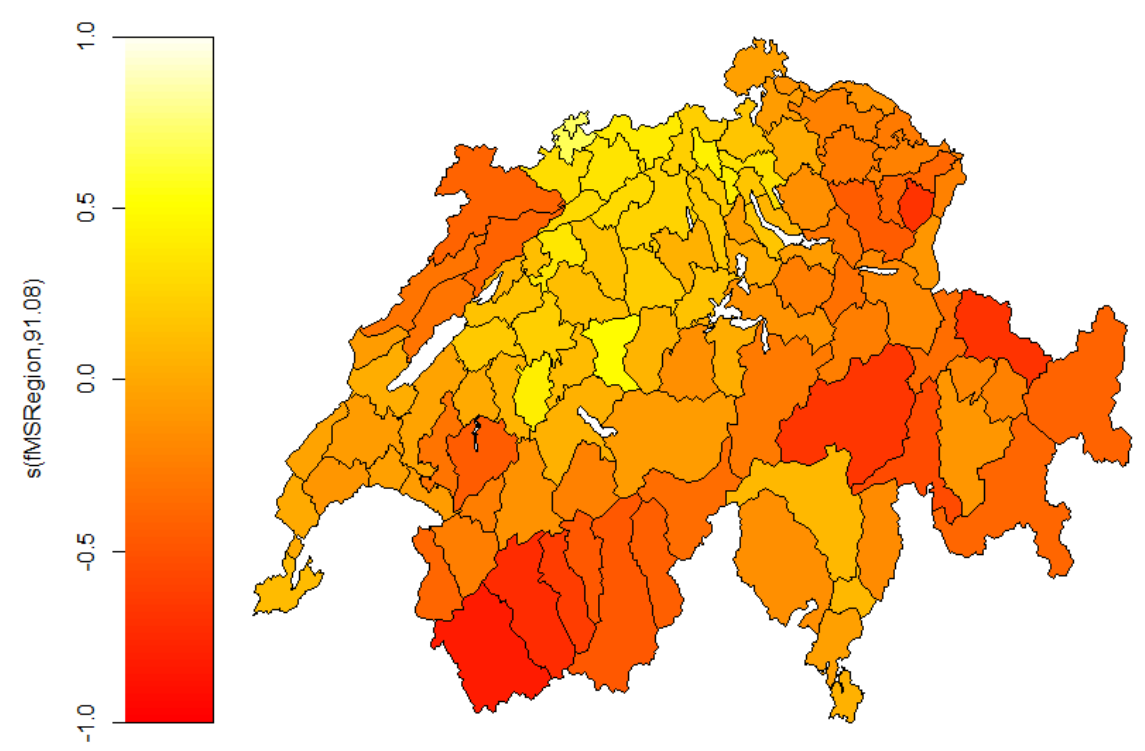

Figure D: Smoothed BMI effect across MS regions 


\section{Results from alternative models}

\subsection{Ordinal regression}

Here, we use ordinal regression formulated as a GAM model with smooth effects similar to those used previously for BMI modelling. The main motivation is to utilize an alternative model (less influenced by potential outliers or other data problems) as a form of sensitivity check.

Fami1y: ordered Categorical $(-1,3.19,4.86,84)$

Link function: identity

Formula:

OBMI $\sim \mathrm{s}($ Year, bs = "cr") + furbanNon. Urban + fAgegroup + fscheme + fISEIGroups + s(Mean_SSEP, bs = "cr") + s(Week_Mon, bs $=$ "cc") $+s($ fMSRegion, bs $=$ "mrf", $x t=x$. MSRegion $)+$ $\mathrm{s}($ post1ockdown 1 , by $=$ zero, bs $=" c r ", p c=0)+s($ post1ockdown 2 , by $=$ zero, bs $=$ "cr", pc $=0$ )

Parametric Terms:

df $\quad$ F p-value

furbanNon.urban $127.631 \quad 1.47 \mathrm{e}-07$

fagegroup $\quad 3508.905<2 e-16$

$\begin{array}{llll}\text { fscheme } & 1 & 2.201 & 0.138\end{array}$

fISEIGroups $\quad 4495.313<2 \mathrm{e}-16$

Approximate significance of smooth terms:

$$
\text { edf Ref.df } \quad F \text { p-value }
$$

$\mathrm{s}($ Year) $\quad 5.174 \quad 6.29513 .051<2 \mathrm{e}-16$

S(Mean_SSEP) $\quad 4.1445 .117124 .832<2 \mathrm{e}-16$

S(Week_Mon) $\quad 5.278 \quad 8.000 \quad 7.267<2 \mathrm{e}-16$

$\mathrm{s}($ fMSRegion) $\quad 87.58799 .96616 .613<2 \mathrm{e}-16$

s(post1ockdown1):zero $2.839 \quad 3.470 \quad 2.658 \quad 0.05172$

s(postlockdown2):zero $\quad \begin{array}{llll}1.005 & 1.017 & 8.182 & 0.00406\end{array}$ 
$>\operatorname{AIC}(02)$

[1] 575938.1

$>\operatorname{summary}(02)$

Fami1y: Ordered Categorical $(-1,3.19,4.86,84)$

Link function: identity

Formula:

OBMI $\sim \mathrm{s}($ Year, bs = "cr") + furbanNon. Urban + fAgegroup +

fscheme + fISEIGroups + s(Mean_SSEP, bs = "cr") + s(Week_Mon, bs $=" c c ")+s($ fMSRegion, bs $=$ "mrf", $x t=x$. MSRegion $)+$ $\mathrm{s}($ post1ockdown 1 , by $=$ zero, bs $=" c r ", p c=0)+s($ post1ockdown 2 , by $=$ zero, bs $=$ "cr", $p c=0$ )

Parametric coefficients:

Estimate std. Error $t$ value $\operatorname{Pr}(>|t|)$

(Intercept)

furbanNon.urbanurban

fAgegroup20.00-20.99

fagegroup21.00-21.99

fAgegroup $<19.00$

fschemeold

fISEIGroupswithout occupation -0.267770

fISEIGroupstertile 1 (low)

fISEIGroupstertile 3 (high)

fISEIGroupsstudents

0.077609

$-0.117048$

$-0.404172$
2.164111
0.020490
0.050210
0.009552
105.61
$<2 \mathrm{e}-16 * * *$
0.166459
0.010043
$5.2571 .47 \mathrm{e}-07 * * *$
0.362823
0.014948
16.575
$<2 \mathrm{e}-16 * * *$ $-0.172659$
0.00938
24.272
$<2 \mathrm{e}-16 * * *$
0.034897
0.023520
1.484
$<2 \mathrm{e}-16 * * *$

$0.026995-9.919<2 \mathrm{e}-16 * * *$

0.010648

$7.2893 .13 \mathrm{e}-13 * * *$

$0.011061-10.582$

$<2 \mathrm{e}-16 * * *$

$0.011235-35.975<2 e-16 * * *$

Signif. codes: 0 “***; 0.001 “**; $0.01 ، * 0.05 ،, 0.1 ،, 1$

Approximate significance of smooth terms:

$$
\text { edf Ref.df } F \text { p-value }
$$


s(Year)

s(Mean_SSEP)

s(Week_Mon)

$s$ (fMSRegion)

s(post lockdown1) : zero

s (post1ockdown2) : zero

\subsection{4}

4.144

5.278

$87.587 \quad 99.966$

$2.839 \quad 3.470$

$1.005 \quad 1.017$
$6.29513 .051<2 \mathrm{e}-16 * * *$

$5.117124 .832<2 \mathrm{e}-16 * * *$

8.000

$7.267<2 \mathrm{e}-16 * * *$

$16.613<2 \mathrm{e}-16 * * *$

2.6580 .05172 .

$8.1820 .00406 * *$

Signif. codes: 0 “***; 0.001 “**, 0.01 ‘*’ 0.05 ‘’ 0.1 ‘ 1

Deviance explained $=1.11 \%$

fREML $=7.03 e+05$ scale est. $=1$

$\mathrm{n}=332320$

\subsection{Heteroscedastic normal regression}

Fami1y: gaulss

Link function: identity logb

Formula:

$\mathrm{BMI} \sim \mathrm{s}($ Year, $\mathrm{bs}=$ "cr") + furbanNon.Urban + fAgegroup + fscheme + fISEIGroups + s(Mean_SSEP, bs = "cr") + s(Week_Mon, bs $=" c c ")+s($ fMSRegion, $b s=$ "mrf", $x t=x$.MSRegion $)+$ $\mathrm{s}($ post1ockdown 1 , by $=$ zero, bs $=" \mathrm{cr} ", \mathrm{pc}=0)+\mathrm{s}($ post1ockdown2, by $=$ zero, bs = "cr", pc =0) $\sim s($ Year, bs $=$ "cr") + furbanNon.urban + fagegroup + fscheme + fISEIGroups + s(Mean_SSEP, bs = "cr") + s(Week_Mon, bs $=" c c ")+s($ fMSRegion, bs $=$ "mrf", $x t=x$.MSRegion $)+$ $\mathrm{s}($ postlockdown1, by $=$ zero, bs $=" \mathrm{cr} ", \mathrm{pc}=0)+\mathrm{s}($ postlockdown2, by $=$ zero, bs $=$ "cr", $p c=0$ )

Parametric Terms:

df Chi.sq p-value 


$\begin{array}{llrr}\text { furbanNon.urban } & 1 & 42.884 & 5.81 \mathrm{e}-11 \\ \text { fAgegroup } & 3 & 2084.781 & <2 \mathrm{e}-16 \\ \text { fscheme } & 1 & 1.523 & 0.217 \\ \text { fISEIGroups } & 4 & 2653.398 & <2 \mathrm{e}-16 \\ \text { furbanNon.Urban.1 } & 1 & 139.207 & <2 \mathrm{e}-16 \\ \text { fAgegroup.1 } & 3 & 1055.304 & <2 \mathrm{e}-16 \\ \text { fscheme.1 } & 1 & 2.471 & 0.116 \\ \text { fISEIGroups.1 } & 4 & 2532.459<2 \mathrm{e}-16\end{array}$

\begin{tabular}{|c|c|c|c|}
\hline & edf & Ref.df & Chi.sq p-value \\
\hline s(Year) & 6.299 & 7.427 & $117.003<2 \mathrm{e}-16$ \\
\hline s(Mean_SSEP) & 4.546 & 5.522 & $806.765<2 e-16$ \\
\hline s(Week_Mon) & 5.717 & 8.000 & $56.932<2 \mathrm{e}-16$ \\
\hline s(fMSRegion) & 90.552 & 101.290 & $2129.421<2 e-16$ \\
\hline s(post1ockdown1): zero & 2.322 & 2.820 & $5.588 \quad 0.11021$ \\
\hline s(post1ockdown2): zero & 3.952 & 4.793 & $15.897 \quad 0.00592$ \\
\hline s.1(Year) & 8.609 & 8.950 & $144.468<2 e-16$ \\
\hline S.1(Mean_SSEP) & 5.011 & 6.017 & $882.793<2 \mathrm{e}-16$ \\
\hline s.1(Week_Mon) & 4.686 & 8.000 & 17.5790 .00115 \\
\hline S.1(fMSRegion) & 89.891 & 100.930 & $1703.167<2 \mathrm{e}-16$ \\
\hline s.1(post1ockdown 1 ): zero & 3.676 & 4.482 & $11.458 \quad 0.02479$ \\
\hline $\begin{array}{l}\text { s.1(post1ockdown2): zero } \\
>\text { summary }(\mathrm{h} 2)\end{array}$ & 3.754 & 4.563 & 12.5140 .02156 \\
\hline
\end{tabular}

Family: gaulss

Link function: identity logb

Formula:

BMI s (Year, bs = "cr") + furbanNon.urban + fagegroup + fscheme + fISEIGroups + s(Mean_SSEP, bs = "cr") + s(Week_Mon, $b s=" c c ")+s($ fMSRegion, $b s=$ "mrf", $x t=x$.MSRegion $)+$ $\mathrm{s}($ post1ockdown 1 , by $=$ zero, bs $=" c r ", p c=0)+s($ postlockdown2, 
by $=$ zero, bs $=$ "cr", pc =0)

$\sim s($ Year, bs $=$ "cr") + furbanNon.urban + fAgegroup + fscheme +

fISEIGroups + s(Mean_SSEP, bs = "cr") + s(Week_Mon,

bs $=" c c ")+s($ fMSRegion, bs = "mrf", $x t=x$.MSRegion $)+$

$\mathrm{s}($ postlockdown 1 , by $=$ zero, bs $=" c r ", p c=0)+s($ postlockdown 2 ,

by $=$ zero, bs $=" c r ", p c=0$ )

Parametric coefficients:

(Intercept)

$*$

furbanNon. Urbanurban

$*$

fAgegroup20.00-20.99

$*$

fAgegroup21.00-21.99

0.753080

$-0.355315$

$0.015528-22.883<2 \mathrm{e}-16 * *$

fAgegroup $<19.00$

0.350243

0.018178

$19.267<2 \mathrm{e}-16 * *$

$*$

fschemeold

0.054605

0.044244

1.234

0.217
fISEIGroupswithout occupation

$*$

fISEIGroupsTertile 1 (low)

$*$

fISEIGroupstertile 3 (high)

$*$

fISEIGroupsStudents

$-0.741893$

$0.018841-39.377<2 \mathrm{e}-16 * *$

$*$

(Intercept) .1

1.340836

$0.007522178 .246<2 \mathrm{e}-16 * *$

$*$

furbanNon. Urbanurban. 1

0.037137

0.003148

$11.799<2 \mathrm{e}-16 * *$

$-0.497002$

$0.046899-10.597<2 \mathrm{e}-16 * *$

$0.01999510 .017<2 \mathrm{e}-16 * *$

$0.019788-10.259<2 \mathrm{e}-16 * *$ $*$

$-0.203014$
$23.4890490 .038207614 .776<2 \mathrm{e}-16 * *$

Estimate std. Error $z$ value $\operatorname{Pr}(>|z|)$ 


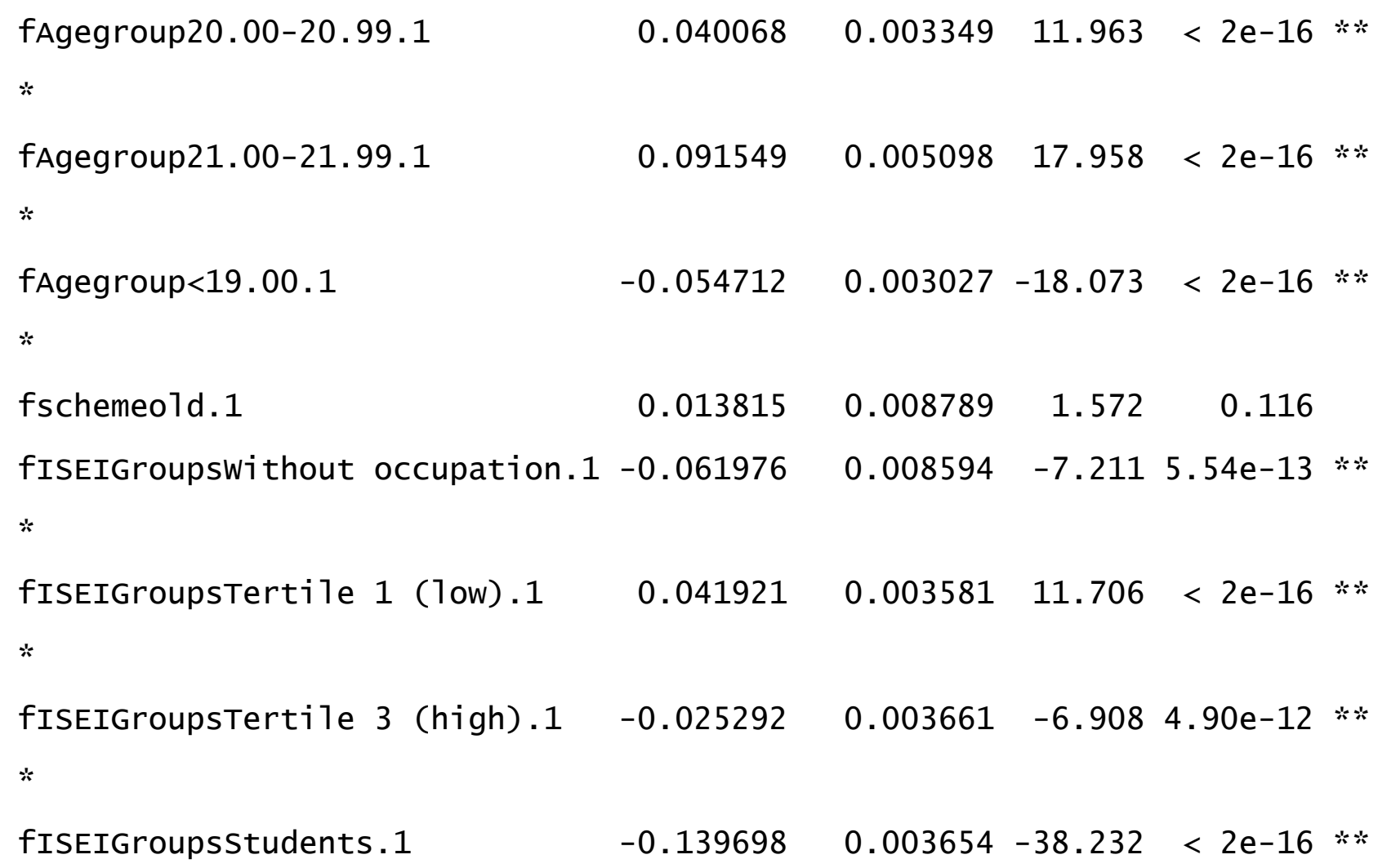


Signif. codes: 0 “***, 0.001 “**, 0.01 ‘*, $0.05 ،, 0.1 ،, 1$

Deviance explained $=2.67 \%$

- REML $=9.1307 \mathrm{e}+05$ Scale est. $=1 \quad \mathrm{n}=332320$

$>\operatorname{AIC}(\mathrm{h} 2)$

[1] 1825621 